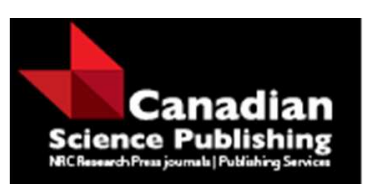

Canadian Journal of Physics

Revue canadienne de physique

\title{
Parameters identification for the unsteady helical flows of a generalized Oldroyd-B fluid model based on its numerical solution
}

\begin{tabular}{|r|l|}
\hline Journal: & Canadian Journal of Physics \\
\hline Manuscript ID & cjp-2016-0853.R1 \\
\hline Manuscript Type: & Article \\
\hline Date Submitted by the Author: & 14-Jan-2017 \\
\hline Complete List of Authors: & $\begin{array}{l}\text { Ma, Ying; Shandong University, School of Mathematics } \\
\text { Jiang, XiaoYun; Shandong Univ, } \\
\text { Yu, Bo; Shandong University, School of Mathematics and Statistics }\end{array}$ \\
\hline Keyword: & $\begin{array}{l}\text { Generalized Oldroyd-B fluid, Riemann-Liouville fractional derivative, } \\
\text { Parameter identification, Implicit difference scheme, Fractional sensitivity } \\
\text { coefficients }\end{array}$ \\
\hline
\end{tabular}

\section{SCHOLARONE ${ }^{\text {m }}$ \\ Manuscripts}




\title{
Parameters identification for the unsteady helical flows of a generalized Oldroyd-B fluid model based on its numerical solution
}

\author{
Ying $\mathrm{Ma}^{1}$, Xiaoyun Jiang ${ }^{1 *}$ and $\mathrm{Bo} \mathrm{Yu}^{2}$
}

\begin{abstract}
In this paper, we mainly consider the problem of parameters identification for the unsteady helical flows of a generalized Oldroyd-B fluid between two infinitely long coaxial circular cylinders. Implicit finite difference method is employed to obtain the numerical solution for the direct problem. By means of the Levenberg-Marquardt method, the numerical inversion for identifying optimal three parameters of the model is implemented simultaneously, that is the Riemann-Liouville timefractional derivative $\alpha$, relaxation time $\lambda$ and retardation time $\lambda_{r}$. In order to testify the validity of the proposed numerical method, varied initial guesses and the observed data whether or not involve random error have been used to verify the reliable of estimation. This paper provides an efficient method to obtain the estimated value of the unknown parameters for the generalized non-Newtonian fluids model.
\end{abstract}

Keywords. Generalized Oldroyd-B fluid, Riemann-Liouville fractional derivative, Parameter identification, Implicit difference scheme, Fractional sensitivity coefficients.

\section{Introduction}

With the development of studies on flows of non-Newtonian fluids and extensive applications in biomechanics, engineering and industry, more and more researchers are interested in modelling non-Newtonian flow phenomena. As a subclass of rate type non-Newtonian fluids, the Oldroyd-B fluid [1-7] which describes the elastic and memory effects exhibited by most biological liquids

\footnotetext{
${ }^{1}$ School of Mathematics, Shandong University, Jinan 250100, PR China.

${ }^{2}$ School of Mathematics and Statistics, Shandong University, Weihai, Weihai 264209, PR China.

${ }^{*}$ Corresponding author. E-mail addresses: wqjxyf@sdu.edu.cn (Xiaoyun Jiang).
} 


\section{Canadian Journal of Physics}

and polymeric as well as stress-relaxation, creep and the normal stress differences that develop during simple shear flow. It has been extensively used in many applications and the results of simulations fit experimental data quite well $[3,7]$. Studies on the motion of Oldroyd-B fluid in cylindrical domains aroused extensive attention. Fetecau et al. [8, 9] and Jamil et al. [10] analyzed the ordinary Oldroyd-B fluid model.

In recent years, as a natural generalization of the classical, fractional calculus has been successfully applied in the constitutive modeling of nonNewtonian fluids. They can simply describe the complex behavior and are better appropriate for describing the complex characteristics of viscoelastic materials. Qi and $\mathrm{Xu}$ [11] discussed the two types of unsteady unidirectional flows of a generalized Oldroyd-B fluid between two parallel plates. Qi and Jin [12] investigated the unsteady helical flow of a generalized Oldroyd-B fluid. Nadeem [13] gave the exact solutions for general periodic flows of fractional Oldroyd-B fluid for an infinite edge. Fetecau et al. [14, 15] studied incompressible generalized Oldroyd-B fluid model. Kang and $\mathrm{Xu}$ [16] investigated the influence of various parameters on the velocity profiles for flow past an accelerated horizontal plate in a rotating fluid with the generalized Oldroyd-B model. Shah et al. [17] obtained the exact analytic solution of a viscoelastic fluid with the generalized Oldroyd-B model. Tong et al. [18] analyzed unsteady helical flows of a generalized Oldroyd-B fluid in concentric cylinders and a circular cylinder. Yang and Zhu [19] considered the thermodynamic compatibility and mechanical analogue of the generalized Oldroyd-B and generalized Jeffreys fluids with fractional derivatives. Similar studies were given in the papers [20-24]. Besides, numerical methods have hold dominant position for solving the constitutive model of the viscoelasticity fluid. Mompean and Deville [25] provided a finite volume technique to simulate the Oldroyd-B fluid through a three-dimensional planar contraction. Vinay et al. [26] examined the numerical simulation of transient non-isothermal flows of a viscoplastic fluid in a pipe. Tomé et al. [27] used finite difference method for simulating viscoelastic axisymmetric free surface flow of an Oldroyd-B fluid.

Nowadays, based on generalized models with fractional derivative, identifying model parameter from experimental data has attracted extensive research. The basic parameters of a fluid can describe the molecular dynamics of the medium and can be used as supplementary parameters describing fluid properties [28], hence the model parameters play a significant role in characterizing the fluid flow phenomena, and the parameters identification is of increasing interest in a number of areas such as engineering, systems, networks, molecular physics, etc. Various methods have been applied to estimate unknown parameters in more and more fields. The Levenberg-Marquardt (LM) method [29] was shown to be efficient to estimate the order of fractional derivative in fractional single-phase-lag heat equation by Ghazizadeh et al. [30]. Zhuang and $\mathrm{Yu}$ [31] estimated the parameter of time-fractional heat conduction in a composite medium by using carbon-carbon experimental data. $\mathrm{Yu}$ and Jiang [32-34] proposed the L-M method to estimate the unknown 
time-fractional orders and the parameters of the generalized models. Chen and Liu [35] considered an inverse problem for identifying two fractional derivatives in a two-dimensional space-fractional nonlocal model with variable diffusivity coefficients. Work by Fan and Jiang [36] applied a Bayesian method to identify three parameters for the fractional fractal diffusion model based on experimental data.

Motivated by the above mentioned works, to the best of our knowledge, there is no literature on the parameters identification for the generalized Oldroyd-B fluid model. The following content of this paper is organized as follows: In section 2, the unsteady helical flows of a generalized Oldroyd-B fluid with fractional derivative model is showed. We give the implicit finite difference scheme for the direct problem in section 3 . In section 4 , we formulate the nonlinear least squares model associated with the inverse problem and give the L-M algorithm for identifying the fractional order, relaxation and retardation times. Results and discussions are analyzed in section 5. Finally in Section 6, some conclusions are drawn based on the numerical results.

\section{Mathematical model}

For an incompressible generalized Oldroyd-B fluid with fractional derivative, Yang and Zhu [19] demonstrated that the generalized Oldroyd-B fluid has a mechanical analogue and is thermodynamically compatible made up of fractional elements if the two orders of fractional derivatives in left and right of the constitutive equations are equal. In the circumstances, it is also required that the retardation time be less than or equal to the relaxation time. The Cauchy stress tensor $\mathbf{T}$ is given by the conservation and constitutive equations $[11-18,20,21]$

$$
\begin{gathered}
\mathbf{T}=-p \mathbf{I}+\mathbf{S} \\
\left(1+\lambda^{\alpha} D_{t}^{\alpha}\right) \mathbf{S}+\lambda\left[(\mathbf{V} \cdot \nabla) \mathbf{S}-\mathbf{L S}-\mathbf{S L}^{T}\right] \\
=\mu\left\{\left(1+\lambda_{r}^{\alpha} D_{t}^{\alpha}\right) \mathbf{A}+\lambda_{r}\left[(\mathbf{V} \cdot \nabla) \mathbf{A}-\mathbf{L} \mathbf{A}-\mathbf{A} \mathbf{L}^{T}\right]\right\},
\end{gathered}
$$

where $p$ is the pressure, $\mathbf{I}$ is the identity tensor, $\mathbf{S}$ is the extra stress tensor, $\mathbf{V}$ is the velocity vector, $\mu$ is the viscosity coefficient, $\lambda$ and $\lambda_{r}$ are relaxation and retardation times, $\alpha$ is fractional calculus parameter. The first RivlinEricksen tensor $\mathbf{A}$ is given by $\mathbf{A}=\mathbf{L}+\mathbf{L}^{T}$, where $\mathbf{L}$ is the velocity gradient tensor, $\mathrm{T}$ indicates the matrix transpose. The fractional differential operator $D_{t}^{\alpha}$ based on Riemann-Liouville's definition [37] is defined as

$$
D_{t}^{\alpha}[f(t)]=\frac{1}{\Gamma(1-\alpha)} \frac{d}{d t} \int_{0}^{t} \frac{f(t)}{(t-z)^{\alpha}} d z, \quad(0 \leq \alpha<1),
$$

in which $\Gamma(\cdot)$ is Gamma Function. While $\alpha=1$, this model can be reduced to the simple Oldroyd-B fluid, if $\lambda=\lambda_{r}=0$, it also includes the classical Newtonian fluid as a special case. While $\lambda=0$ it describes the generalized second grade fluid, if $\lambda_{r} \rightarrow 0$, this model can be simplified as the fractional Maxwell fluid. 


\section{Canadian Journal of Physics}

The incompressible fluid undergoes only isochoric motion, hence $\operatorname{div} \mathbf{V}=$ 0 . In cylindrical coordinates $(r, \theta, z)$, the helical flow velocity is given by

$$
\mathbf{V}=\mathbf{V}(r, t)=v(r, t) \mathbf{e}_{\theta}+w(r, t) \mathbf{e}_{\mathbf{z}}
$$

in which $\mathbf{e}_{\theta}$ and $\mathbf{e}_{z}$ are the unit vectors in the $\theta$ - and $z$-directions, $r$ is the radius of circular cylinders. $v(r, t)$ and $w(r, t)$ are the velocity field in the $\theta$ and $z$-directions. Since the velocity field $\mathbf{V}$ is independent of $\theta$ and $z$, we assume that $\mathbf{S}$ is independent of $\theta$ and $z$. Considering the initial condition $\mathbf{S}(r, 0)=\mathbf{0}$ that $S_{\mathrm{rr}}=0$, in the absence of body forces and a pressure gradient in the axial direction, Eq. (2.2) and the balance of linear momentum [12, 16 , 20] lead to governing equations for the unsteady helical flows of a generalized Oldroyd-B fluid with time-fractional derivative (UHF-GOBF) model

$$
\begin{array}{r}
\left(1+\lambda^{\alpha} D_{t}^{\alpha}\right) \frac{\partial}{\partial t} v(r, t)=\nu\left(1+\lambda_{r}^{\alpha} D_{t}^{\alpha}\right)\left(\frac{\partial^{2}}{\partial r^{2}}+\frac{1}{r} \frac{\partial}{\partial r}-\frac{1}{r^{2}}\right) v(r, t), \\
\left(1+\lambda^{\alpha} D_{t}^{\alpha}\right) \frac{\partial}{\partial t} w(r, t)=\nu\left(1+\lambda_{r}^{\alpha} D_{t}^{\alpha}\right)\left(\frac{\partial^{2}}{\partial r^{2}}+\frac{1}{r} \frac{\partial}{\partial r}\right) w(r, t),
\end{array}
$$

where $\nu=\mu / \rho$ is the kinematic viscosity of fluid. The initial and boundary conditions are

$$
\begin{gathered}
v(r, 0)=\partial_{t} v(r, 0)=0, w(r, 0)=\partial_{t} w(r, 0)=0, \quad a<r<b, \\
v(a, t)=\varphi(t), v(b, t)=\theta(t), w(a, t)=\psi(t), w(b, t)=\phi(t), \quad 0 \leq \mathrm{t} \leq T .
\end{gathered}
$$

\section{Finite difference method for the direct problem}

In this section, we aim to solve the UHF-GOBF (2.5)-(2.8) by finite difference method. Firstly, in a two-dimensional domain $[a, b] \times[0, T]$, we define a uniform grid of mesh point $\left(r_{j}, t_{k}\right)$, where $r_{j}=a+j h, t_{k}=k \tau, j=0,1, \ldots M, k=$ $0,1, \ldots N$ with $h=(b-a) / M, \tau=T / N, M, N \in \mathrm{Z}^{+}$are the spatial and temporal mesh size, respectively. $v_{\mathrm{j}}^{k}$ and $w_{\mathrm{j}}^{k}$ are denoted as numerical solutions at the mesh point $\left(r_{j}, t_{k}\right), V_{\mathrm{j}}^{k}$ and $W_{\mathrm{j}}^{k}$ are denoted as exact solutions.

Then, using the relationship between the Grünwald-Letnikov and the Riemann-Liouville fractional derivatives [37, 38], we have

$$
{ }_{0} D_{t}^{\alpha} f(t)=\lim _{\tau \rightarrow 0} \tau^{-\alpha} \sum_{l=0}^{[t / \tau]}(-1)^{l}\left(\begin{array}{c}
\alpha \\
l
\end{array}\right) f(t-l \tau) \approx \tau^{-\alpha} \sum_{l=0}^{k} c_{l} f(t-l \tau),
$$

where $c_{l}=(-1)^{l} \alpha \cdot(\alpha-1) \cdots(\alpha-l+1) / l !, l=0,1,2 \cdots k$.

Finally, we obtain the implicit finite difference schemes for the UHFMGOBF (2.5)-(2.8): 


$$
\begin{gathered}
\frac{v_{j}^{k}-v_{j}^{k-1}}{\tau}+\frac{\lambda^{\alpha}}{\tau^{\alpha}} \sum_{l=0}^{k} c_{l} \frac{v_{j}^{k-l}-v_{j}^{k-l-1}}{\tau}=\nu\left[\frac{\delta_{x}^{2} v_{j}^{k}}{h^{2}}+\frac{v_{j+1}^{k}-v_{j}^{k}}{(a+j h) h}-\frac{v_{j}^{k}}{(a+j h)^{2}}\right] \\
+\frac{\nu \lambda_{r}^{\alpha}}{\tau^{\alpha}} \sum_{l=0}^{k} c_{l}\left[\frac{\delta_{x}^{2} v_{j}^{k-l}}{h^{2}}+\frac{v_{j+1}^{k-l}-v_{j}^{k-l}}{(a+j h) h}-\frac{v_{j}^{k-l}}{(a+j h)^{2}}\right] \\
\frac{w_{j}^{k}-w_{j}^{k-1}}{\tau}+\frac{\lambda^{\alpha}}{\tau^{\alpha}} \sum_{l=0}^{k} c_{l} \frac{w_{j}^{k-l}-w_{j}^{k-l-1}}{\tau}=\nu\left[\frac{\delta_{x}^{2} w_{j}^{k}}{h^{2}}+\frac{w_{j+1}^{k}-w_{j}^{k}}{(a+j h) h}\right] \\
\quad+\frac{\nu \lambda_{r}^{\alpha}}{\tau^{\alpha}} \sum_{l=0}^{k} c_{l}\left[\frac{\delta_{x}^{2} w_{j}^{k-l}}{h^{2}}+\frac{w_{j+1}^{k-l}-w_{j}^{k-l}}{(a+j h) h}\right] \\
v_{j}^{0}=0, \frac{v_{j}^{0}-v_{j}^{-1}}{\tau}=0, w_{j}^{0}=0, \frac{w_{j}^{0}-w_{j}^{-1}}{\tau}=0, \quad j=0,1, \ldots M \\
v_{0}^{k}=\varphi\left(t_{k}\right), v_{M}^{k}=\theta\left(t_{k}\right), w_{0}^{k}=\psi\left(t_{k}\right), w_{M}^{k}=\phi\left(t_{k}\right), \quad k=0,1, \ldots N, \quad
\end{gathered}
$$

\section{Levenberg-Marquardt algorithm for the parameters identification}

In this section, we revisit an iterative method which is Levenberg-Marquardt method for the inverse problem involving the estimation of unknown three parameters such as Riemann-Liouville time-fractional derivative, relaxation time and retardation time. Set $\mathbf{p}=\left(\alpha, \lambda, \lambda_{r}\right)^{T}$, then the parameter identification of $\mathbf{p}$ is transformed to solving the following nonlinear least squares problem:

$$
\min F(\mathbf{p})=(\mathbf{Y}-\mathbf{U})^{T}(\mathbf{Y}-\mathbf{U})=\frac{1}{2} \sum_{i=1}^{M}\left\{\left(y_{1 i}-v_{i}\right)^{2}+\left(y_{2 i}-w_{i}\right)^{2}\right\},
$$

where $\mathbf{U}=\left\{\left(v_{1}, v_{2}, \cdots, v_{M}\right)^{T},\left(w_{1}, w_{2}, \cdots, w_{M}\right)^{T}\right\}$ is a vector of estimated velocity and $\mathbf{Y}=\left\{\left(y_{11}, y_{12}, \cdots, y_{1 M}\right)^{T},\left(y_{21}, y_{22}, \cdots, y_{2 M}\right)^{T}\right\}$ is a vector of observed data, $y_{1 i}$ and $y_{2 i}$ are observed data for velocity in the $\theta$ - and $z$ directions, here both of vectors are at some time $t=t_{k}$ and radius $r=r_{j}$.

The sensitivity matrix $[29,35]$ plays a fundamental role in the parameter estimation, its sensitivity coefficients can be computed by finite difference approximation, we have

$$
\mathbf{J}^{k}=\left(\mathbf{J}_{\alpha}^{k}, \mathbf{J}_{\lambda}^{k}, \mathbf{J}_{\lambda_{r}}^{k}\right), \quad \mathbf{J}_{\xi_{i}}^{k}=\frac{\mathbf{v}\left(\cdot, \mathbf{p}^{k}+\delta \mathbf{e}_{i}\right)-\mathbf{v}\left(\cdot, \mathbf{p}^{k}\right)}{\delta},
$$

where $\delta$ is the numerical differential step, $\mathbf{e}_{1}=(1,0,0)^{T}, \mathbf{e}_{2}=(0,1,0)^{T}, \mathbf{e}_{3}=$ $(0,0,1)^{T}, i=1,2,3$.

L-M method is an improved method based on Gauss-Newton method [29] which overcomes the rank-deficient of sensitivity matrix and converges 


\section{Canadian Journal of Physics}

fast with the requirement of a good initial guess. The L-M iterative scheme for identify the three parameters of the model is equivalent to minimize the least squares norm Eq. (4.1). The iteration for unknown parameter $\mathbf{p}$ is then given by [35]:

$$
\mathbf{p}^{k+1}=\mathbf{p}^{k}-\left(\left(\mathbf{J}^{k}\right)^{T} \cdot \mathbf{J}^{k}+\mu^{k} \mathbf{I}\right)^{-1}\left(\mathbf{J}^{k}\right)^{T}(\mathbf{Y}-\mathbf{U}),
$$

The stopping criterion $[31,32,35]$ of the iterative algorithm is

$$
\left\|\mathbf{p}^{k+1}-\mathbf{p}^{k}\right\|<\varepsilon
$$

here $\|\cdot\|$ is the vector Euclidean norm.

Then implementation of the L-M algorithm for identifying three unknown parameters can be summarized as follows:

step 1. Use implicit finite difference method to obtain the solution of direct problem (2.5)-(2.8). Set $\mathbf{U}\left(\cdot, \mathbf{p}_{*}\right)$ as the final observation data, $\mathbf{p}_{*}$ as the true value of the parameter.

step 2. Calculate the damping parameter $\mu^{k}=F\left(\mathbf{p}^{k}\right)$ from Eq. (4.1).

step 3. Compute the Jacobian sensitivity matrix $\mathbf{J}^{k}$.

step 4. If it satisfies the stopping criteria (4.4), then stop.

step 5 . Replace $k$ by $k+1$. Calculate the new estimation $\mathbf{p}^{k+1}$ which defined in Eq. (4.3), then return step 2 refresh the damping parameter $\mu^{k+1}$, and continue the rest of iteration steps.

\section{Results and discussions}

\subsection{Example 1}

Consider the following equation

$$
\begin{gathered}
\left(1+\lambda^{\alpha} D_{t}^{\alpha}\right) \frac{\partial}{\partial t} v(r, t)=0.165\left(1+\lambda_{r}^{\alpha} D_{t}^{\alpha}\right)\left(\frac{\partial^{2}}{\partial r^{2}}+\frac{1}{r} \frac{\partial}{\partial r}-\frac{1}{r^{2}}\right) v(r, t), \\
\left(1+\lambda^{\alpha} D_{t}^{\alpha}\right) \frac{\partial}{\partial t} v(r, t)=0.165\left(1+\lambda^{\alpha} D_{t}^{\alpha}\right)\left(\frac{\partial^{2}}{\partial r^{2}}+\frac{1}{r} \frac{\partial}{\partial r}\right) w(r, t),
\end{gathered}
$$

with the initial and boundary conditions

$$
\begin{aligned}
& v(r, 0)=\partial_{t} v(r, 0)=0, w(r, 0)=\partial_{t} w(r, 0)=0, \quad 1<r<2, \\
& v(1, t)=2, v(2, t)=2, w(1, t)=2, w(2, t)=2, \quad 0 \leq \mathrm{t} \leq 2 .
\end{aligned}
$$

In this subsection, firstly we verify the correctness of the numerical solution by fitting the exact solution curve in Ref. [18] to our numerical solution and analyse the effects of different parameters for the flow. Secondly we apply the L-M method to obtain the numerical inversions for the parameters identification by using the numerical solution as the available observed data and analyze the relationship of the sensitivity coefficients for three parameters. Finally we obtain the results by using the available data with random error, and compare the estimation results with final observed data which contain random error. 


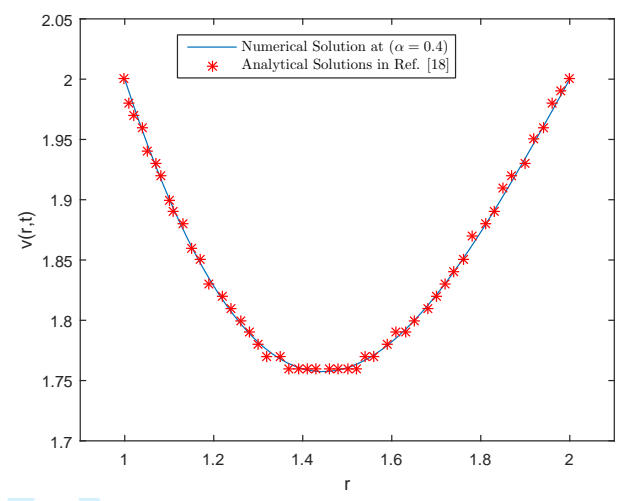

FIG. 1. Shows the comparison between numerical solutions and analytical solutions of the velocity profiles $v(r, t)$ at $\alpha=0.4$.

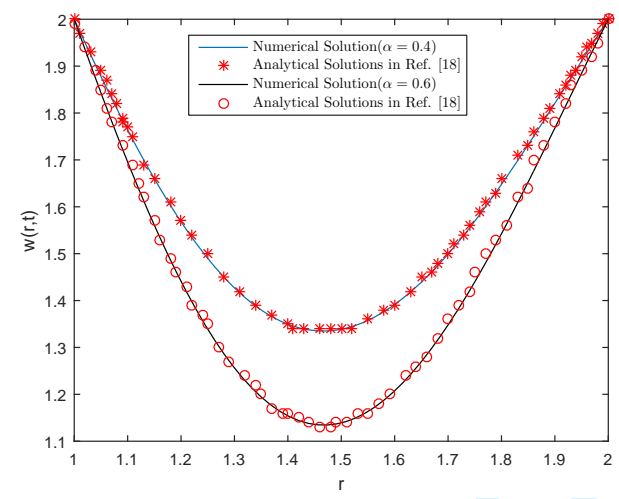

Fig. 2. Shows the comparison between numerical solution$\mathrm{s}$ and analytical solutions of the velocity profiles $w(r, t)$ at $\alpha=0.4$ and $\alpha=0.6$.

\subsubsection{Validation of the numerical solution.}

Tong et al. [18] obtained exact solutions of some unsteady helical flows of Oldroyd-B fluid in an annular pipe by using Hankel transform and Laplace transform for fractional calculus and analyzed the influence of the orders of the fractional parameter on the velocity profiles. In Figs. 1 and 2, the numerical solutions in this paper are extremely consistent with the analytical solutions from Ref. [18] at $T=2, \nu=0.165, \alpha=0.4,0.6, \lambda=15, \lambda_{r}=8$. The curve fitting results of the velocity in two directions indicate that the numerical solutions by implicit finite difference method are accurate.

To observe the effects of different parameters for the flow, in Fig. 3, the variations of the velocity field $v(r, t)$ is plotted for different of $\alpha$ with 


\section{Canadian Journal of Physics}

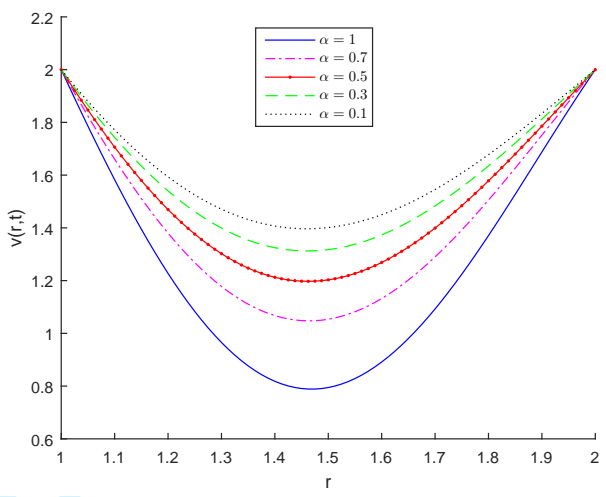

FIG. 3. Velocity profiles $v(r, t)$ versus $r$ for different fractional orders $\alpha$.

$T=2, M=N=80, \lambda=7, \lambda_{r}=3$ and it is the ordinary model when $\alpha=1$. Similarly in Fig. 4 , the velocity profiles against $r$ depending on $\lambda$ with $T=2, M=N=80, \alpha=0.5, \lambda_{r}=8$ and the velocity profiles against $r$ depending on $\lambda_{r}$ with $T=2, M=N=80, \alpha=0.5, \lambda=15$. In Ref. $[12,18]$ the effects for two orders of fractional derivatives in left and right of the constitutive equations are contrary on the velocity. In this paper, the combine effects of the fractional order parameters on the velocity $v(r, t)$ are shown in Fig. 3, we can obviously find out that the curves are higher when $\alpha$ is smaller and lower when $\alpha$ is larger, and it means the smaller the $\alpha$, the smaller the velocity changes. From Fig. 4 we can find the effects of the relaxation and retardation times are totally opposite, the velocity is increased when $\lambda$ is smaller or $\lambda_{r}$ is larger. Thus, it is obvious that the order of the fractional derivative, the relaxation time and retardation time have strong effects on the velocity field.

\subsubsection{The parameters identification for the available data without random error.}

Denote $\mathbf{p}_{0}=\left(\alpha_{0}, \lambda_{0}, \lambda_{r 0}\right)^{T}$ as the initial guess, $\mathbf{p}_{*}$ as the true value of the parameter to be estimated and $\mathbf{p}_{i n v}$ as the result of parameter estimation. To verify the efficient of the L-M algorithm, we use

$$
e_{r r}=\frac{\left\|\mathbf{p}_{i n v}-\mathbf{p}_{*}\right\|_{\max }}{\left\|\mathbf{p}_{*}\right\|_{\max }},
$$

to measure the relative error between the true value and the estimated result.

Analyzing the relationship between the sensitivity coefficients [39] of three parameters can provide a reference for choosing the method of the parameters identification. If they are linear correlation, it will be needed to estimate three parameters one by one. Otherwise, they can be estimated together. We introduce the expression of relative sensitivity coefficients as 


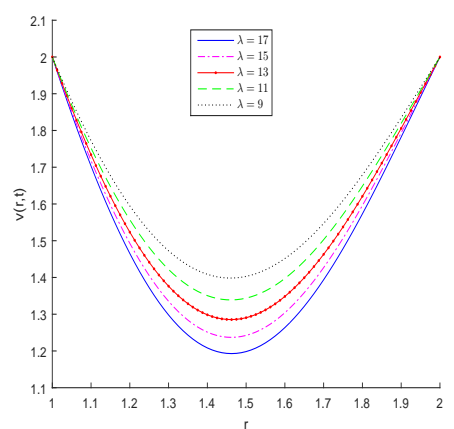

(a) Effect of $\lambda$

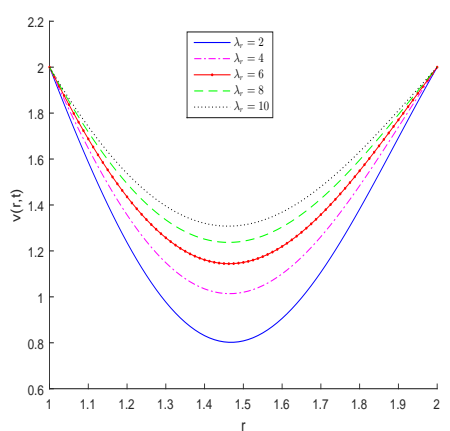

(b) Effect of $\lambda_{r}$

FIG. 4. Velocity profiles $v(r, t)$ versus $r$ for different parameters $\lambda$ and $\lambda_{r}$.

follows

$$
J_{\xi_{i}}=\frac{\xi_{i}}{U_{\text {mean }}} \cdot \frac{\partial \mathbf{U}}{\partial \xi_{i}}, \quad(i=1,2,3),
$$

where $\xi_{i}\left(\xi_{1}=\alpha, \xi_{2}=\lambda, \xi_{2}=\lambda_{r}\right)$. $\mathbf{U}$ is a vector of computed velocity and $U_{\text {mean }}$ is its arithmetic mean.

For the direct problem, we take $M=20, N=100, T=2$. For the inverse problem, we choose step as $\delta=10^{-5}$ and tolerance as $\varepsilon=10^{-6}$. Table 1 lists the identified results of the parameters, the relative error and the number of iterations for different initial guesses when the true value of the parameter $\mathbf{p}_{*}^{T}=(0.5,7,3),(0.5,4,2)$, respectively. We observe that the deviation made by initial guess and the true value mainly affects the number of iterations but has little influence on the final estimation. Fig. 5 shows the sensitivity coefficients $\mathbf{J}_{\alpha}, \mathbf{J}_{\lambda}, \mathbf{J}_{\lambda_{r}}$ of the velocity in two directions at $\mathbf{p}_{*}^{T}=(0.5,4,2), \mathbf{p}_{0}^{T}=(0.9,10,4)$. Both of them are linearly independent, illustrating that it is reasonable to estimate the three unknown parameters at the same time.

\subsubsection{The parameters identification for the available data with random er- ror.}

In the actual application, the available data we can use are always containing some random measurement error [32], here we define

$$
u_{\text {mea }}=u_{\text {exa }}(1+\eta) \text {, }
$$

where $u_{\text {mea }}$ is the final observed data with random perturbation item $u_{\text {exa }} \cdot \eta$, $u_{\text {exa }}$ is the solution for the problem.

Table 2 lists the parameters identification results for different initial guesses and the available data with random measurement error. From Table 2 , we can obviously find out the random measurement error influenced the accuracy of the estimation and number of iterations, but for a fixed random 


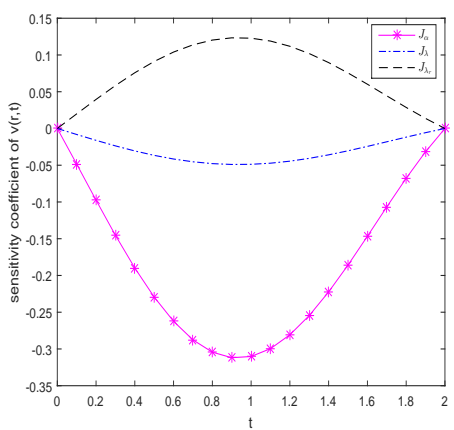

(a) velocity $v(r, t)$

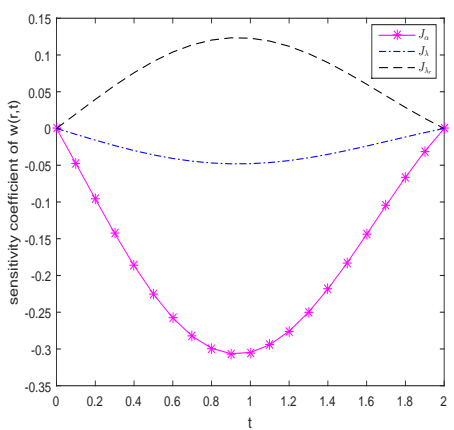

(b) velocity $w(r, t)$

FIG. 5. Sensitivity coefficients for $\alpha, \lambda$ and $\lambda_{r}$.

TABLE 1 . The effects of the initial guess $\mathbf{p}_{0}^{T}$ on the parameter identification results at $\mathbf{p}_{*}^{T}=(0.5,7,3),(0.5,4,2)$.

\begin{tabular}{lccc}
\hline $\mathbf{p}_{0}^{T}$ & $\mathbf{p}_{i n v}^{T}$ & $e_{r r}$ & Iter \\
\hline$(0.2,4,2)$ & $(0.500002673,7.000030211,3.000035308)$ & $5.0440 \mathrm{e}-06$ & 22 \\
$(0.4,6,5)$ & $(0.500006725,7.000067156,3.000080154)$ & $1.1451 \mathrm{e}-05$ & 18 \\
$(0.7,8,8)$ & $(0.500000340,7.000025725,3.000014210)$ & $3.6750 \mathrm{e}-06$ & 27 \\
$(0.8,16,9)$ & $(0.500000009,7.000000224,3.000000153)$ & $3.2008 \mathrm{e}-08$ & 102 \\
$(0.9,10,1)$ & $(0.500000069,7.000001031,3.000000974)$ & $1.4735 \mathrm{e}-07$ & 24 \\
\hline$(0.2,2,1)$ & $(0.500000010,4.000000129,2.000000115)$ & $3.2362 \mathrm{e}-08$ & 16 \\
$(0.4,6,5)$ & $(0.500000080,4.000000987,2.000000870)$ & $2.4678 \mathrm{e}-07$ & 19 \\
$(0.7,8,8)$ & $(0.500008363,4.000103790,2.000090937)$ & $2.5948 \mathrm{e}-05$ & 46 \\
$(0.8,15,7)$ & $(0.499999877,4.000007432,2.000002384)$ & $1.8579 \mathrm{e}-06$ & 148 \\
$(0.9,10,4)$ & $(0.499999900,4.000006251,2.000002015)$ & $1.5626 \mathrm{e}-06$ & 38 \\
\hline
\end{tabular}

measurement error, different initial guesses have little effect on the final results. Fig. 6 demonstrates the comparison of the true value $\mathbf{p}_{*}^{T}=(0.5,7,3)$, measurement data with random error $\eta=0.01 \%, 0.1 \%$ and estimated results. We can observe that the estimated results are in good agreement with the corresponding available data. Based on the above analysis, L-M method is efficient for identifying the parameters of the unsteady helical flows of a generalized Oldroyd-B fluid model. 
TABle 2. Parameters identification results $\left(\mathbf{p}_{*}^{T}=(0.5,7,3)\right)$ for various initial guesses $\mathbf{p}_{0}^{T}$ and different random errors $\eta$.

\begin{tabular}{lccc}
\hline$p_{0}^{T}$ & $\eta$ & $\mathbf{p}_{\text {inv }}^{T}$ & Iter \\
\hline$(0.2,4,1)$ & $0.01 \%$ & $(0.525578487,6.915645046,3.133082860)$ & 22 \\
$(0.8,15,8)$ & $0.01 \%$ & $(0.526788371,6.924206053,3.145526409)$ & 80 \\
$(0.2,4,1)$ & $0.03 \%$ & $(0.578108334,6.627656961,3.299324174)$ & 35 \\
$(0.8,15,8)$ & $0.03 \%$ & $(0.579868817,6.632032149,3.311677626)$ & 97 \\
$(0.2,4,1)$ & $0.05 \%$ & $(0.633323773,6.245287988,3.361215208)$ & 49 \\
$(0.8,15,8)$ & $0.05 \%$ & $(0.634237334,6.253746984,3.370621747)$ & 112 \\
$(0.2,4,1)$ & $0.07 \%$ & $(0.689942550,5.821462434,3.340065155)$ & 58 \\
$(0.8,15,8)$ & $0.07 \%$ & $(0.689657717,5.822593380,3.339693264)$ & 141 \\
$(0.2,4,1)$ & $0.1 \%$ & $(0.777377212,5.182094762,3.210175921)$ & 71 \\
$(0.8,15,8)$ & $0.1 \%$ & $(0.777531935,5.181442439,3.210170447)$ & 189 \\
\hline
\end{tabular}

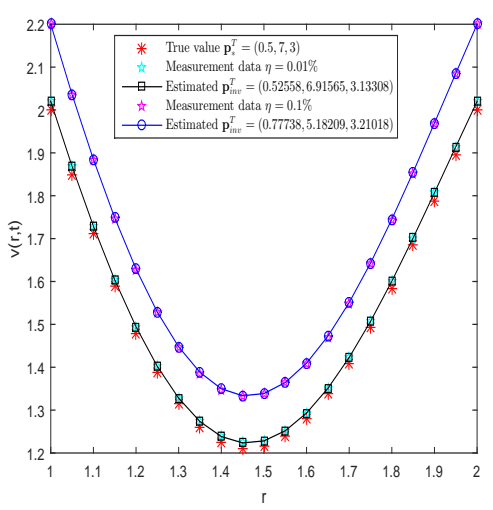

(a) velocity $v(r, t)$

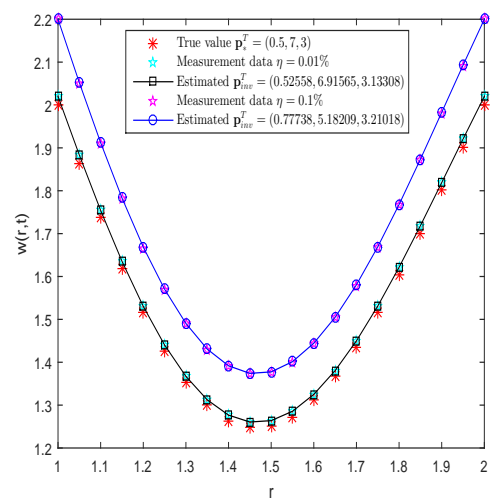

(b) velocity $w(r, t)$

FIG. 6. Comparison of the exact solution $\mathbf{p}_{*}^{T}=(0.5,7,3)$, measurement data $(\eta=0.01 \%, 0.1 \%)$ and estimated result.

\subsection{Example 2}

Consider the following equation with the source term

$$
\begin{aligned}
& \left(1+\lambda^{\alpha} D_{t}^{\alpha}\right) \frac{\partial}{\partial t} v(r, t)=\left(1+\lambda_{r}^{\alpha} D_{t}^{\alpha}\right)\left(\frac{\partial^{2}}{\partial r^{2}}+\frac{1}{r} \frac{\partial}{\partial r}-\frac{1}{r^{2}}\right) v(r, t) \\
& +e^{r} t^{3 \alpha+1}\left\{\left[(3 \alpha+2)+\frac{\lambda^{\alpha} \Gamma(3 \alpha+3)}{t^{\alpha} \Gamma(2 \alpha+2)}\right]-\left(1+\frac{1}{r}-\frac{1}{r^{2}}\right) t\left[1+\frac{\lambda_{r}^{\alpha} \Gamma(3 \alpha+3)}{t^{\alpha} \Gamma(2 \alpha+3)}\right]\right\}
\end{aligned}
$$




\section{Canadian Journal of Physics}

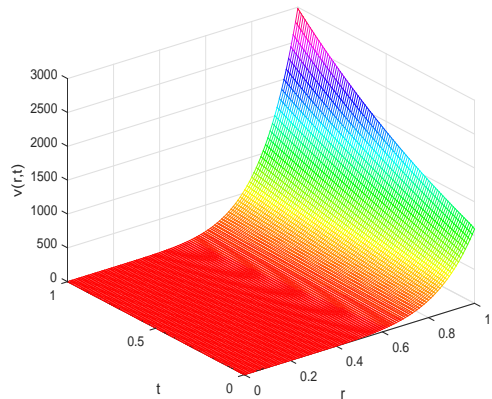

(a) Numerical results

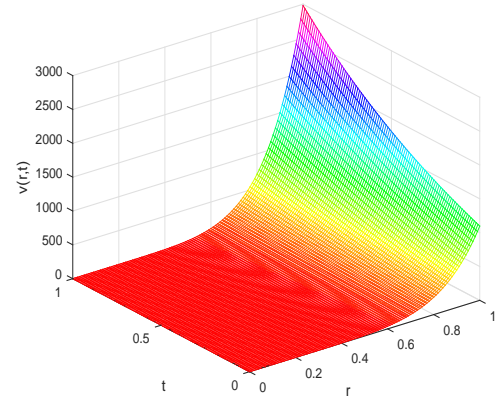

(b) Exact results

FIG. 7. Solution for velocity $v(r, t)$ at $T=1, M=N=$ $100, \alpha=0.5, \lambda=7, \lambda_{r}=3$.

$$
\begin{aligned}
& \left(1+\lambda^{\alpha} D_{t}^{\alpha}\right) \frac{\partial}{\partial t} v(r, t)=\left(1+\lambda_{r}^{\alpha} D_{t}^{\alpha}\right)\left(\frac{\partial^{2}}{\partial r^{2}}+\frac{1}{r} \frac{\partial}{\partial r}\right) w(r, t) \\
& +e^{r} t^{4 \alpha+2}\left\{\left[(4 \alpha+3)+\frac{\lambda^{\alpha} \Gamma(4 \alpha+4)}{t^{\alpha} \Gamma(3 \alpha+3)}\right]-\left(1+\frac{1}{r}\right) t\left[1+\frac{\lambda_{r}^{\alpha} \Gamma(4 \alpha+4)}{t^{\alpha} \Gamma(3 \alpha+4)}\right]\right\},
\end{aligned}
$$

with the initial and boundary conditions

$$
\begin{aligned}
& v(r, 0)=\partial_{t} v(r, 0)=0, w(r, 0)=\partial_{t} w(r, 0)=0, \quad 0<r<1, \\
& v(0, t)=e^{\lambda} t^{3 \alpha+\lambda_{r}+2}, \quad v(1, t)=e^{\lambda+1} t^{3 \alpha+\lambda_{r}+2}, \\
& w(0, t)=e^{\lambda} t^{4 \alpha+\lambda_{r}+3}, \quad w(1, t)=e^{\lambda+1} t^{4 \alpha+\lambda_{r}+3}, \quad 0 \leq \mathrm{t} \leq 1 .
\end{aligned}
$$

The exact solution is

$$
v(r, t)=e^{r+\lambda} t^{3 \alpha+\lambda_{r}+2}, \quad w(r, t)=e^{r+\lambda} t^{4 \alpha+\lambda_{r}+3} .
$$

In this subsection, we use the L-M method to obtain the parameter identification results in two cases, the available data involved and not involved random measurement error.

\subsubsection{The parameters identification for the available data without random} error.

Let $T=1, M=20, N=100, \mathbf{p}_{*}^{T}=(0.5,7,3), \delta=10^{-6}, \varepsilon=10^{-5}$. Table 3 lists the estimation results of three parameters for the velocity $v(r, t)$ and $w(r, t)$ with different initial guesses. Results implied the initial guess has little influence on the final estimation. The three-dimensional pictures of the exact and numerical solutions of velocity $v(r, t)$ and $w(r, t)$ are presented in Figs. 7 and 8 , respectively. Fig. 9 gives the sensitivity coefficients of the velocity field which indicates it is reasonable to estimate the three unknown parameters simultaneously. 
TABLE 3. The effects of the initial guess $\mathbf{p}_{0}^{T}$ on the parameter identification results.

\begin{tabular}{ccccc}
\hline $\mathbf{p}_{0}^{T}$ & $\mathbf{p}_{*}$ & $\mathbf{p}_{i n v}$ & $e_{r r}$ & Iter \\
\hline$(0.2,4,2)$ & 0.5 & 0.500000000000074 & $3.6809 \mathrm{e}-13$ & 27 \\
& 7 & 7.000000000000003 & & \\
& 3 & 2.999999999997423 & & \\
$(0.3,6,7)$ & 0.5 & 0.499999999999014 & $1.8361 \mathrm{e}-12$ & 23 \\
& 7 & 7.000000000000004 & & \\
& 3 & 3.000000000012852 & & \\
$(0.6,15,8)$ & 0.5 & 0.499999999999660 & $1.2872 \mathrm{e}-13$ & 44 \\
& 3 & 7.000000000000002 & & \\
& 7 & 3.000000000000901 & & \\
$(0.8,9,9)$ & 0.5 & 0.500000000000446 & $7.3770 \mathrm{e}-13$ & 44 \\
& 7 & 6.999999999999998 & & \\
$(0.9,10,1)$ & 0.5 & 2.999999999994836 & & \\
& 7 & 7.009999999999860 & $5.5353 \mathrm{e}-13$ & 15 \\
& 3 & 2.999999999996125 & & \\
\hline
\end{tabular}

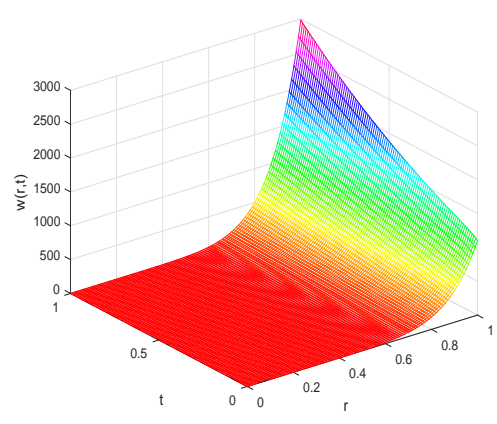

(a) Numerical results

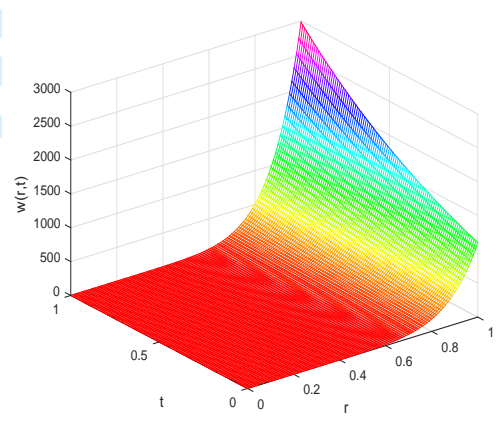

(b) Exact results

FIG. 8. Solution for velocity $w(r, t)$ at $T=1, M=N=$ $100, \alpha=0.5, \lambda=7, \lambda_{r}=3$.

\subsubsection{The parameters identification for the available data with random er- ror. \\ From Table 4, we can obviously find out that the estimation results are consistent with final observed data contained random error. In Table 5, under the data with different random error, we take maximum relative errors between 4 groups of estimation results in various initial guess, we can see that differences of the estimation results are proportional to random error.}




\section{Canadian Journal of Physics}

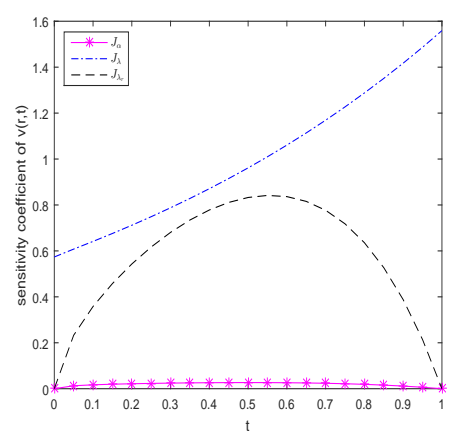

(a) velocity $v(r, t)$

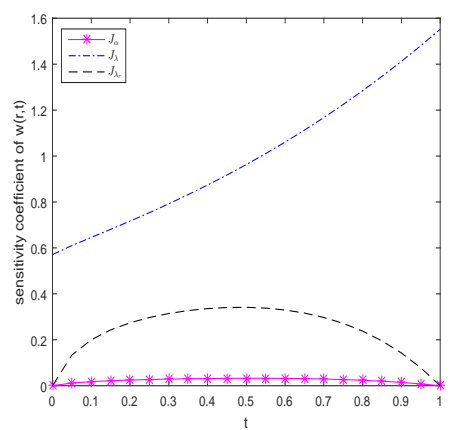

(b) velocity $w(r, t)$

FIG. 9. Sensitivity coefficients for $\alpha, \lambda$ and $\lambda_{r}$.

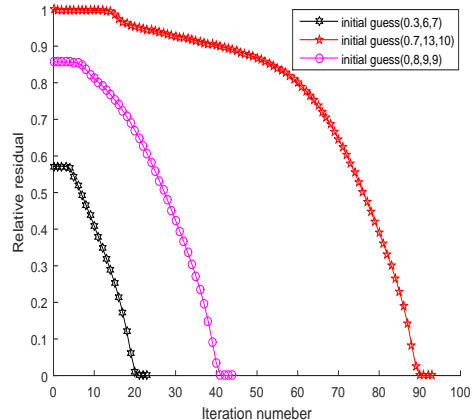

(a) The available data without error

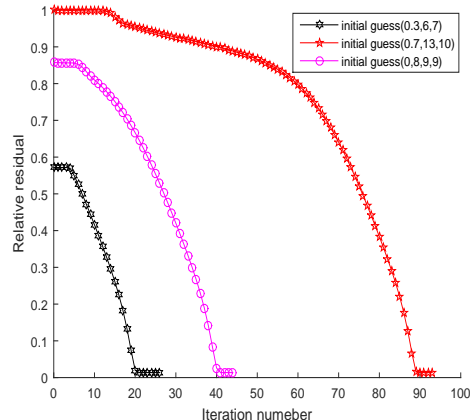

(b) The available data with $10 \%$ error

FIG. 10. Convergence behaviors of the L-M method for estimating fractional parameters.

Fig. 10 shows the convergence behavior of L-M method for the accurate data and data with $10 \%$ error by plotting the iteration number corresponding to the relative residual. From Fig. 10, we can see that L-M method applied in parameter identification presents diverse convergence behaviors in different initial guesses. Besides, the closer the initial guess approaches to the actual value, the fewer iteration required to obtain the final estimation result. Based on the above analysis, L-M method is reliable and effective for identifying the unknown parameters of the generalized non-Newtonian fluid model.

\section{Conclusions}

In this paper, we consider the problem of parameters identification for the unsteady helical flow of a generalized Oldroyd-B fluid with Riemann-Liouville 
TABle 4. Parameters identification results for various initial guesses $\mathbf{p}_{0}^{T}$ and different random errors $\eta$.

\begin{tabular}{lccc}
\hline$p_{0}^{T}$ & $\eta$ & $\mathbf{p}_{\text {inv }}^{T}$ & Iter \\
\hline$(0.2,4,1)$ & $2 \%$ & $(0.4994895197,7.0198017529,3.0023743141)$ & 29 \\
$(0.4,6,5)$ & $2 \%$ & $(0.4994895197,7.0198017529,3.0023743140)$ & 14 \\
$(0.6,10,2)$ & $2 \%$ & $(0.4994895198,7.0198017529,3.0023743133)$ & 14 \\
$(0.9,16,9)$ & $2 \%$ & $(0.4994895197,7.0198017529,3.0023743150)$ & 58 \\
$(0.2,4,1)$ & $4 \%$ & $(0.4989918643,7.0392189857,3.0046828976)$ & 29 \\
$(0.4,6,5)$ & $4 \%$ & $(0.4989918642,7.0392189857,3.0046828992)$ & 14 \\
$(0.6,10,2)$ & $4 \%$ & $(0.4989918647,7.0392189857,3.0046828930)$ & 14 \\
$(0.9,16,9)$ & $4 \%$ & $(0.4989918643,7.0392189857,3.0046828973)$ & 57 \\
$(0.2,4,1)$ & $10 \%$ & $(0.4975703444,7.0953060114,3.0112440834)$ & 31 \\
$(0.4,6,5)$ & $10 \%$ & $(0.4975703446,7.0953060114,3.0112440812)$ & 14 \\
$(0.6,10,2)$ & $10 \%$ & $(0.4975703444,7.0953060114,3.0112440834)$ & 14 \\
$(0.9,16,9)$ & $10 \%$ & $(0.4975703450,7.0953060114,3.0112440754)$ & 57 \\
$(0.2,4,1)$ & $16 \%$ & $(0.4962457891,7.1484135570,3.0173134408)$ & 33 \\
$(0.4,6,5)$ & $16 \%$ & $(0.4962457891,7.1484135570,3.0173134416)$ & 15 \\
$(0.6,10,2)$ & $16 \%$ & $(0.4962457894,7.1484135570,3.0173134372)$ & 15 \\
$(0.9,16,9)$ & $16 \%$ & $(0.4962457901,7.1484135570,3.0173134277)$ & 57 \\
$(0.2,4,1)$ & $20 \%$ & $(0.4954109662,7.1823136690,3.0211167589)$ & 35 \\
$(0.4,6,5)$ & $20 \%$ & $(0.4954109661,7.1823136690,3.0211167603)$ & 16 \\
$(0.6,10,2)$ & $20 \%$ & $(0.4954109668,7.1823136690,3.0211167514)$ & 14 \\
$(0.9,16,9)$ & $20 \%$ & $(0.4954109659,7.1823136690,3.0211167636)$ & 61 \\
\hline
\end{tabular}

TABLE 5. The relative error of parameters identification for data accompanied with different random errors.

\begin{tabular}{lccccc}
\hline$\eta$ & $2 \%$ & $4 \%$ & $10 \%$ & $16 \%$ & $20 \%$ \\
\hline$e_{r r}$ & $2.8288 \mathrm{e}-3$ & $5.6027 \mathrm{e}-3$ & $1.3615 \mathrm{e}-2$ & $2.1202 \mathrm{e}-2$ & $2.6045 \mathrm{e}-2$ \\
\hline
\end{tabular}

time-fractional derivative between two infinitely long coaxial circular cylinders. The implicit finite difference scheme is employed to solve the generalized Oldroyd-B fluid model. Then based on numerical solution, we use the L-M method to identify the time-fractional derivative $\alpha$, relaxation time $\lambda$ and retardation time $\lambda_{r}$. Results indicate that various initial guesses have little effect on the final estimation and the estimation results are consistent with final observed data which contain random error or not contain. Therefore, the L-M method is efficient and reliable in identifying the parameters for the generalized non-Newtonian fluid with fractional derivative model.

This paper provides a valid parameter identification method for the unsteady helical flows of a generalized Oldroyd-B fluid model, which can be applied in the flow of other complex viscoelastic fluids model. 


\section{Canadian Journal of Physics}

\section{Acknowledgments}

We wish to thank the referees for their many constructive comments which improved the present paper, and we would like to thank Prof. Haijun Su and Prof. Haitao Qi for their thoughtful suggestions. The work is supported by the National Natural Science Foundation of China (Grants Nos.11472161, 11102102 and 91130017) and China Postdoctoral Science Foundation (Grant No. 2016M602127).

\section{References}

[1] J.G. Oldroyd. Proc. R. Soc. Lond. A. 200, 523 (1950).

[2] J.G. Oldroyd. I. Q. J. Mech. Appl. Math. 4(3), 271 (1951).

[3] J.G. Oldroyd. Proc. R. Soc. Lond. A. 245(1241), 278 (1958).

[4] H.A. Attia. Can. J. Phys. 81(5), 789 (2003).

[5] S. Saravanan. Cana. J. Phys. 85(9), 947 (2007).

[6] T. Haroon, A.M. Siddiqui, and M. Zeb. Can. J. Phys. 93(8), 871 (2015).

[7] G. Zaman, S. Islam, Y.H. Kang, and I.H. Jung. Sci. China Phys. Mech. $55(1), 125$ (2012).

[8] C. Fetecau, T. Hayat, and C. Fetecau. J. Non-Newton. Fluid Mech. 153, 191 (2008).

[9] C. Fetecau, M. Imran, C. Fetecau, and I. Burdujan. ZAMP. 61(5), 959 (2010).

[10] M. Jamil, C. Fetecau, and M. Imran. Comm. Non-Linear Sci. Numer. Simul. 16(3), 1378 (2011).

[11] H.T. Qi and M.Y. Xu. Appl. Math. Model. 33(11), 4184 (2009).

[12] H.T. Qi and H. Jin. Nonlinear Anal. RWA. 10(5), 2700 (2009).

[13] S. Nadeem. Phys. Lett. A. 368(3), 181 (2007).

[14] C. Fetecau, C. Fetecau, M. Kamran, and D. Vieru, J. Non-Newton. Fluid Mech. 56(3), 189 (2009).

[15] D. Vieru, C. Fetecau, and C. Fetecau. Can. J. Phys. 88(9), 675 (2010).

[16] J.H. Kang and M.Y. Xu. Acta Mech. Sinica. 25(4), 463 (2009).

[17] S.H.A.M. Shah, M. Khan, and H.T. Qi. Nonlinear Anal. RWA. 10(4), $2590(2009)$.

[18] D.K. Tong, X.M. Zhang, and X.H. Zhang. J. Non-Newton. Fluid Mech. 156(1), 75 (2009).

[19] P. Yang and K.Q. Zhu. SCI. China Phys. Mech. 54(4), 737 (2011).

[20] M. Nazar, Q. Sultan, M. Athar, and M. Kamran. Comm. Non-Linear Sci. Numer. Simul. 16(7), 2737 (2011).

[21] C.R. Li, L.C. Zheng, Y. Zhang, L.X. Ma, and X.X. Zhang. Comm. NonLinear Sci. Numer. Simul. 17(12), 5026 (2012).

[22] M. Nazar, A. Mahmood, M. Athar, and M. Kamran. Chem. Eng. Commu. 199(2), 290 (2012).

[23] M. Kamran, M. Imran, and M. Athar. Meccanica. 48(5), 1215 (2013).

[24] M. Jamil, N.A. Khan, and M.A. Imran. Int. J. Nonlin. Sci. Num. 14(7), 443 (2013). 
[25] G. Mompean and M. Deville. J. Non-Newton. Fluid Mech. 72(2), 253 (1997).

[26] G. Vinay, A. Wachs, and J.F. Agassant. J. Non-Newton. Fluid Mech. 128(2), 144 (2005).

[27] M.F. Tomé, L. Grossi, A. Castelo, J.A. Cuminato, S. McKee, and K. J. Walters. J. Non-Newtonian. Fluid Mech. 141(2), 148 (2007).

[28] B.S. Tripathi, A. Awasthi, and A. Awasthi. Can. J. Phys. 88(4), 283 (2010).

[29] M.N. Özisik. Inverse heat transfer: fundamentals and applications. Boca Raton: CRC Press, 2000.

[30] H.R. Ghazizadeh, A. Azimi, and M. Maerefat. Int. J. Heat Mass Tran. 55(7), 2095 (2012).

[31] Q. Zhuang, B. Yu, and X.Y. Jiang. Physica B. 456, 9 (2015).

[32] B. Yu, X.Y. Jiang, and H.T. Qi. Acta Mech. Sinica. 31(2), 153 (2015).

[33] B. Yu, X.Y. Jiang, and C. Wang. Appl. Math. Comput. 274106 (2016).

[34] B. Yu and X.Y. Jiang. J. Sci. Comput. 68(1) 252 (2016).

[35] S.Z. Chen, F.W. Liu, X.Y. Jiang, I. Turner, and K. Burrage. SIAM J. Numer. Anal. 54(2), 606 (2016).

[36] W.P. Fan, X.Y. Jiang, and S.Z. Chen. Comput. Math. Appl. 71(2), 642 (2016).

[37] I. Podlubny. Fractional Differential Equations. Academic Press, New York. 1999.

[38] C.M. Chen, F.W. Liu, I. Turner, and V. Anh. J. Comput. Phys. 227(2), 886 (2007).

[39] N.A. Malik, R.A. Ghanam, and S. Al-Homidan. Can. J. Phys. 93(1), 18 (2014).

Ying $\mathrm{Ma}^{1}$

Xiaoyun Jiang ${ }^{1 *}$

$\mathrm{Bo} \mathrm{Yu}^{2}$ 